\title{
Human umbilical vein endothelial cell vaccine suppresses the angiogenesis of esophageal squamous cell carcinoma in a humanized mouse model
}

\author{
HANGFAN LIU $^{1 *}$, JIMIN ZHAO $^{1,2^{*}}$, YI YANG $^{1}$, GUOGUO JIN $^{1}$, XIAOYAN ZHANG $^{1,2}$, \\ DONGYU WANG ${ }^{1}$, CHAO XIE $^{1}$, KANGDONG LIU ${ }^{1,2}$, XINHUAN CHEN $^{1,2}$, \\ XIANG LI $^{1,2}$, FANG TIAN ${ }^{1,2}$, ZIMING DONG ${ }^{1,2}$ and JING LU ${ }^{1,2}$ \\ ${ }^{1}$ Department of Pathophysiology, Basic Medical College, Zhengzhou University; \\ ${ }^{2}$ Collaborative Innovation Center of Henan Province for Cancer Chemoprevention, Zhengzhou, Henan 450001, P.R. China
}

Received February 14, 2018; Accepted August 6, 2018

DOI: $10.3892 /$ or.2018.6653

\begin{abstract}
The antitumor effect of the human umbilical vein endothelial cell (HUVEC) vaccine has been well documented; however, its anti-angiogenic effects on human esophageal squamous cell carcinoma (ESCC) have yet to be studied. In the present study, a 'humanized' mouse model was established by transplanting NOD/SCID mice with human peripheral blood mononuclear cells (PBMC). After 4 weeks, the level of cluster of differentiation (CD)- $45^{+}$human T-lymphocytes in mouse peripheral blood was $>0.1 \%$, which indicated that mouse reconstruction and the human immune system transformation had been successful. The humanized mice were used to evaluate the anti-angiogenic effect of the HUVEC vaccine on human ESCC. After immunization with the HUVEC vaccine for 5 consecutive weeks, the humanized mice were subcutaneously transplanted with EC9706 cells. The results indicated that the HUVEC vaccine reduced the size of human esophageal carcinoma xenografts by suppressing angiogenesis. In addition, the HUVEC-immunized mice exhibited reduced expression of angiogenesis-associated antigens (vascular endothelial growth factor receptor 2 and VE-Cadherin) in the tumor specimens, and increased levels of angiogenesis-associated antibodies in the serum. Notably, the HUVEC vaccine also increased the infiltration of human T-lymphocytes into the spleen of humanized mice. In conclusion, the present study demonstrated the anti-angiogenic effect of the HUVEC vaccine on ESCC in a
\end{abstract}

Correspondence to: Dr Jing Lu, Department of Pathophysiology, Basic Medical College, Zhengzhou University, 100 Science Road, Zhengzhou, Henan 450001, P.R. China

E-mail: lujing@zzu.edu.cn

"Contributed equally

Key words: human umbilical vein endothelial cell, esophageal squamous cell carcinoma, humanized mouse model, angiogenesisassociated antigen humanized mouse model, and set an experimental foundation for the application of the HUVEC vaccine in ESCC patients.

\section{Introduction}

Esophageal carcinoma is the eighth most common and most severe malignant tumor worldwide (1). Despite the different therapeutic regimens employed with patients, including surgical resections, radiotherapy and chemotherapy, the survival rate has continued to be low for many decades. There are two strategies associated with cancer immunotherapy: one strategy induces or enhances immune-mediated tumor cell destruction, and the other counteracts tumor cell evasion from the host's immune system or boosts immune-mediated destruction (2). In our previous study, dendritic cells were used as the basis of a tumor vaccine for stimulating the immune system to target the antigens associated with the tumor (3). It has been well established that angiogenesis forms the basis of tumor development and metastasis; thus, suppressing tumor-associated angiogenesis has become a favorable strategy for the treatment of cancer (4). Active immunization with a whole endothelial cell vaccine can control tumor size and metastasis by restraining tumor angiogenesis in mice (5). Human umbilical vein endothelial cell (HUVEC) vaccines have been effective in anti-angiogenesis immunity against melanoma, glioblastoma, colorectal cancer and hepatocellular carcinoma (5-8). However, the anti-angiogenic effect of the HUVEC vaccine on ESCC is still unknown. Xenograft mouse models have often been used to evaluate the anticancer effect in ESCC. Transplantable tumors in immunocompetent mice can be used to design therapeutic strategies and analyze the underlying immunological mechanisms; however, the value of these animal models has often been questioned when in comparison with patients (9). As a substitute, reconstituted mouse models, which consist of immunodeficient mice that are repopulated with human immune cells, are promising alternatives (10). In the past few decades, modified transplantation models, which support the transplantation and progression of a human hematolymphoid system, have become a significant experimental technique within this field of research (11). 
NOD/SCID mice are thought to be ideal models for immunodeficient and immune reconstruction studies (12). A previous study also demonstrated that NOD/SCID mice transplanted with hominine hematopoietic tissue or peripheral blood mononuclear cells (PBMC) could reconstitute a functional immune system (13). Considering the role of the HUVEC vaccine in antitumor and anti-angiogenic activities in many types of cancers, the present study engrafted human PBMCs into NOD/SCID mice to reconstruct a humanized immune system in order to evaluate the antitumor and anti-angiogenic impact of the HUVEC vaccine on ESCC, and further investigate the underlying mechanism.

\section{Materials and methods}

Mice and cells. A total of 30 female NOD/SCID mice, aged 4-5 weeks, weighing 18-22 g, were acquired from Vital River Company (Beijing, China). All mice used in the subsequent experiments were treated and observed according to the guidelines of Zhengzhou University Animal Ethics Committee (Henan, China). The animals were housed under specific pathogen-free conditions (light on from 6:00 am to $6: 00 \mathrm{pm}$, temperature $20-26^{\circ} \mathrm{C}$, relative humidity: $40-60 \%$ ) and allowed access to a commercial mice chow and sterilized water ad libitum. The mice were sacrificed by carbon dioxide euthanasia at the 16th week after the initiation of the experiment, and the tumor volume of the mice should not exceed $1,000 \mathrm{~mm}^{3}$. Total murine serum immunoglobulin (Ig) was detected by ELISA in all mice and only non-leaky mice were used for analyses. The esophageal tumor cell line EC9706 was cultured in Dulbecco's modified Eagle's medium containing 10\% fetal bovine serum (FBS) (both from Biological Industries, Kibbutz Beit Haemek, Israel) with 5\% $\mathrm{CO}_{2}$ at $37^{\circ} \mathrm{C}$. The primary HUVECs were separated from the umbilical cord, and cultured with endothelial cell media, as previously described (14).

Quantitation of IgG levels in mice. The NOD/SCID mice blood samples with heparin were centrifuged at $800 \mathrm{x} \mathrm{g}$ for $10 \mathrm{~min}$ and the isolated serum samples were stored until subsequent use. The contents of mouse IgG was detected using an IgG ELISA kit (Arigo, Taiwan, China). The specimens were determined in triplicate by spectrophotometry at an absorbance of $450 \mathrm{~nm}$. According to the standards of Jackson Laboratory (Bar Harbor, ME, USA), an IgG content $<1 \mu \mathrm{g} / \mathrm{ml}$ indicated no immune leakage; these mice were termed the non-leaky mice.

Immune reconstruction of mice. The present study collected human PBMCs from healthy volunteers as described in our previous study (15). Written informed consent was obtained from healthy volunteers. The PBMCs were separated by density gradient centrifugation at $1,500 \mathrm{x} \mathrm{g}$ for $20 \mathrm{~min}$ with lymphocyte medium (TBD Sciences, Tianjin, China). Non-leaky NOD/SCID mice were randomly divided into 2 groups: i) the vehicle group $(n=8)$; and ii) the PBMC group $(n=16)$. The mice in the PBMC group were engrafted with PBMCs $\left(4 \times 10^{7}\right.$ cells/mouse) intraperitoneally. After 4 weeks, the expression of cluster of differentiation (CD) $-45^{+}$T-lymphocytes in the peripheral blood (obtained from the retroorbital venous plexus) of the mice was detected via flow cytometry. When the level of CD $45^{+} \mathrm{T}$-lymphocytesin the peripheral blood was $>0.1 \%$, the immune reconstruction of the mice was considered successful (16).

Flow cytometry. The mouse blood samples were obtained from the retroorbital venous plexus. Single-cell suspensions were generated from engrafted mice spleens and filtered by $70-\mu \mathrm{m}$ filter paper. Blood $(100 \mu \mathrm{l})$ or single-cell splenic suspension $\left(1 \times 10^{6}\right)$ was treated with $10 \mu \mathrm{l}$ anti-human fluorescein isothiocyanate (FITC)-conjugated CD45 antibody (cat. no. ab134199) or FITC-conjugated mouse IgG1 isotype control (cat. no. ab91356; both from Abcam, Cambridge, UK) in the dark for $30 \mathrm{~min}$ at $4^{\circ} \mathrm{C}$. Before washing stained cells with PBS, all of the erythrocytes were treated with FACS lysing solution (BD Biosciences, San Jose, CA, USA) and then resuspended with $1 \mathrm{ml}$ PBS containing $2 \%$ FBS. Finally, T-lymphocytes were analyzed by flow cytometry.

Vaccination protocols in mice. Subconfluent HUVECs were trypsinized, centrifuged, fixed with $0.025 \%$ glutaraldehyde and then suspended in PBS prior to storage at $-80^{\circ} \mathrm{C}$. The successfully immune reconstituted mice (humanized mice) were randomly allocated into 2 groups $(n=8)$ : i) the PBMC group; and ii) the PBMC+HUVEC group. The mice in the PBMC + HUVEC group were immunized 5 times with the HUVEC vaccine $\left(5 \times 10^{6}\right.$ cells/mouse) in the axillary lymphonodus area, once a week. The PBMC group was processed in the same manner using PBS instead. Then the mice were subcutaneously injected with EC9706 cells (6x10\%/mouse) into their left flank. After the tumor became palpable, the size was measured every other day using vernier caliper. The long and short diameters were measured, and the volume of the tumor (V) was calculated using the following formula: $\mathrm{V}\left(\mathrm{mm}^{3}\right)=0.5 \mathrm{x}$ length $\mathrm{x}$ width ${ }^{2}$.

Immunohistochemistry. Tumor tissues and spleens resected from mice were fixed in $4 \%$ paraformaldehyde, then embedded in paraffin for subsequent sectioning. Each sample was cut into $4-\mu \mathrm{m}$ sections and incubated with the primary antibody anti-CD31 overnight at $4{ }^{\circ} \mathrm{C}$ (1:50 dilution; cat. no. ab28364; Abcam) in order to estimate the microvascular content. The number of stained vessels were quantified using microvessel density (MVD). Another section was incubated with mouse anti-human CD45 antibody (1:100 dilution; cat. no. ab187271; Abcam) overnight at $4^{\circ} \mathrm{C}$ in order to analyze the infiltration of the spleen, which was then further incubated with rabbit anti-mouse antibody at room temperature for 2 h (1:1,000 dilution; cat. no. sc-358914; Santa Cruz Biotechnology, Inc., Dallas, TX, USA). Following this, the sections were stained with diaminobenzidine (ZSGB-BIO; OriGene Technologies, Inc., Beijing, China) and hematoxylin. The sections were then observed under an optical microscope (magnification, x200).

Hemoglobin assay. A hemoglobin assay was conducted as previously described (3). Briefly, the amount of hemoglobin in tumors was verified using Drabkin's reagent (Sigma-Aldrich; Merck KGaA, Darmstadt, Germany) according to the manufacturer's instructions. An equal quantity of tumor tissues 
was homogenized in $1 \mathrm{ml}$ oxidizing agent, and the samples were centrifuged at $12,000 \mathrm{x}$ g for $20 \mathrm{~min}$. The samples were then detected at an absorbance of $540 \mathrm{~nm}$ in a spectrophotometer (Thermo Fisher Scientific, Inc., Waltham, MA, USA) after incubation at $37^{\circ} \mathrm{C}$ for $30 \mathrm{~min}$. The relative contents of hemoglobin in tumor specimens were then compared with the vehicle group.

Reverse transcription-quantitative polymerase chain reaction (RT-qPCR). RT-qPCR was performed to determine the changes in vascular endothelial growth factor receptor 2 (VEGFR2) and CD144 mRNA levels. Total RNA from tumor samples was extracted with TRIzol (Invitrogen; Thermo Fisher Scientific Inc.), and cDNA was synthesized with a RT-qPCR kit (Takara Bio, Inc., Otsu, Japan). Target mRNA was quantified using the following primer pairs: GAPDH forward, 5'-GAAGGC TGGGGCTCATTT-3' and reverse, 5'-GAGGAGGCATTG CTGATGAT-3'; VEGFR2 forward, 5'-GAGTGAGGAAGG AGGACGAAGG-3' and reverse, 5'-CCCGTAGGATGATGA CAAGAAGTAGC-3'; CD144 forward, 5'-AAACACCTCACT TCCCCATC-3' and reverse, 5'-ACCTTGCCCACATATTCT CC-3'. The qPCR thermocycling conditions were as follows: Initial denaturation at $95^{\circ} \mathrm{C}$ for $10 \mathrm{~min}$, followed by 40 cycles of denaturation at $95^{\circ} \mathrm{C}$ for $10 \mathrm{sec}$ and annealing at $60^{\circ} \mathrm{C}$ for $30 \mathrm{sec}$. Experiments were repeated 3 times for each sample. The 7500 Fast Real-Time PCR System v2.0.5 (Applied Biosystems; Thermo Fisher Scientific, Inc.) was used to process the results.

Western blot analysis. The tumor tissues were homogenized with Tissue Protein Extraction Reagent (Boster Biological Technology, Co., Ltd., Wuhan, China) to detect protein expression. The lysates were processed and protein content was measured by bicinchoninic acid assay (BCA; Beyotime Institute of Biotechnology, Shanghai, China). Then, $50 \mu \mathrm{g}$ protein of each group was isolated by $10 \%$ SDS-PAGE and transferred to polyvinylidene difluoride membranes (PVDF; EMD Millipore, Billerica, MA, USA) and subsequently incubated in blocking buffer (5\% skimmed milk, $1 \%$ Tween-20 in $20 \mathrm{mmol} / 1$ Tris-buffered saline) at room temperature for $2 \mathrm{~h}$. Then, the PVDF membranes were incubated with anti-VEGFR2 (1:200 dilution; cat. no. ab42228) or anti-CD144 (1:500 dilution; cat. no. ab166715) (both from Abcam) primary antibodies overnight at $4^{\circ} \mathrm{C}$, followed by incubation with rabbit anti-mouse horseradish peroxidase (HRP)-conjugated secondary antibody (1:1,000 dilution; cat. no. sc-358914; Santa Cruz Biotechnology, Inc.) at room temperature for $2 \mathrm{~h}$. In addition, the blots detected by enhanced chemiluminescence kit (Invitrogen; Thermo Fisher Scientific Inc.) according to the manufacturer's instructions. Equal loading of protein was confirmed by probing with monoclonal mouse anti-human $\beta$-actin antibody (1:1,000 dilution; cat. no. sc-130065; Santa Cruz Biotechnology Inc.).

HUVEC membrane protein was prepared using the Membrane Protein Extraction Kit (Beyotime Institute of Biotechnology, Shanghai, China) to detect antibody levels. An equal quantity of proteins was separated and then transferred to the membranes. The membranes were incubated overnight with mouse serum obtained from the mice, and then incubated with a rabbit anti-human secondary antibody labeled with horseradish peroxidase (cat. no. ab6759; Abcam). The results were finally quantified with ImageJ software (National Institutes of Health, Bethesda, MD, USA).

Detection of the anti-HUVEC antibody. ELISAs were performed in order to determine the levels of anti-HUVEC antibody in the serum of mice, as described in our previous study (3). Briefly, HUVEC membrane proteins were loaded onto ELISA plates (JetBioFil, Guangzhou, China). Then the plates were incubated with $100 \mu \mathrm{l}$ serum (1:50), which was obtained from mice the following day. The ELISA was conducted with 3,3',5,5'-tetramethyl benzidine (Beijing Solarbio Science \& Technology Co., Ltd., Beijing, China) and the reaction was terminated using $\mathrm{H}_{2} \mathrm{SO}_{4}$ after incubation with rabbit anti-mouse HRP-conjugated IgG (1:1,000 dilution; cat. no. sc-358914; Santa Cruz Biotechnology, Inc.) at $37^{\circ} \mathrm{C}$ for $2 \mathrm{~h}$, and the optical density at an absorbance of $450 \mathrm{~nm}$ was measured. Each sample was evaluated in 3 separate experiments.

Statistical analysis. Quantitative data was expressed as the mean \pm standard deviation. Student's t-test (two-sided) was conducted for analysis between two subgroups. Statistical significance between $>2$ subgroups were evaluated by one-way analysis of variance followed with Bonferroni and Tamhane post hoc tests. GraphPad Prism (GraphPad Software, Inc., La Jolla, CA, USA) was used for all statistical tests. $\mathrm{P}<0.05$ was considered to indicate a statistically significant difference.

\section{Results}

Establishment of the humanized mouse model. Total murine serum IgG was determined by ELISA. The results indicated that the total murine serum IgG was $<1 \mu \mathrm{g} / \mathrm{ml}$ in all 24 tested mice, which indicated that all of the mice had no immune leakage (Fig. 1A). The non-leaky mice were then used to establish the humanized mouse model.

The levels of human CD $45^{+}$T-lymphocytes in mouse peripheral blood and spleens were assessed by flow cytometry at different time-points after PBMC administration. The peripheral blood levels of human CD45 ${ }^{+}$T-lymphocytes in the PBMC group was $>0.1 \%$ at 4 weeks after PBMC administration $(\mathrm{P}<0.01$; Fig. 1B), which further increased to $>5 \%$ after 14 weeks post-PBMC application $(\mathrm{P}<0.001$; Fig. 1C). Furthermore, the level of human CD $45^{+}$T-lymphocytes in the mouse spleens of the PBMC group was significantly higher when compared with the vehicle group at 14 weeks after the PBMC injections $(\mathrm{P}<0.01$; Fig. 1D).

To further assess the establishment of the humanized engraftment mouse model, the present study examined the recruitment of T-lymphocytes in the spleen 14 weeks after the PBMC injections. The mouse spleens were excised and then weighed; the results revealed that the size and weight of the spleens in the PBMC group were significantly greater when compared with the control group $(\mathrm{P}<0.01$; Fig. $2 \mathrm{~A}$ and $\mathrm{B})$. Then, the expression of the human CD45 leukocyte common antigen in the spleens was analyzed by immunohistochemical staining. The results demonstrated that the spleens of the PBMC group of mice exhibited extensive human CD $45^{+}$T-lymphocyte levels, while the vehicle group did not present CD45 positive 

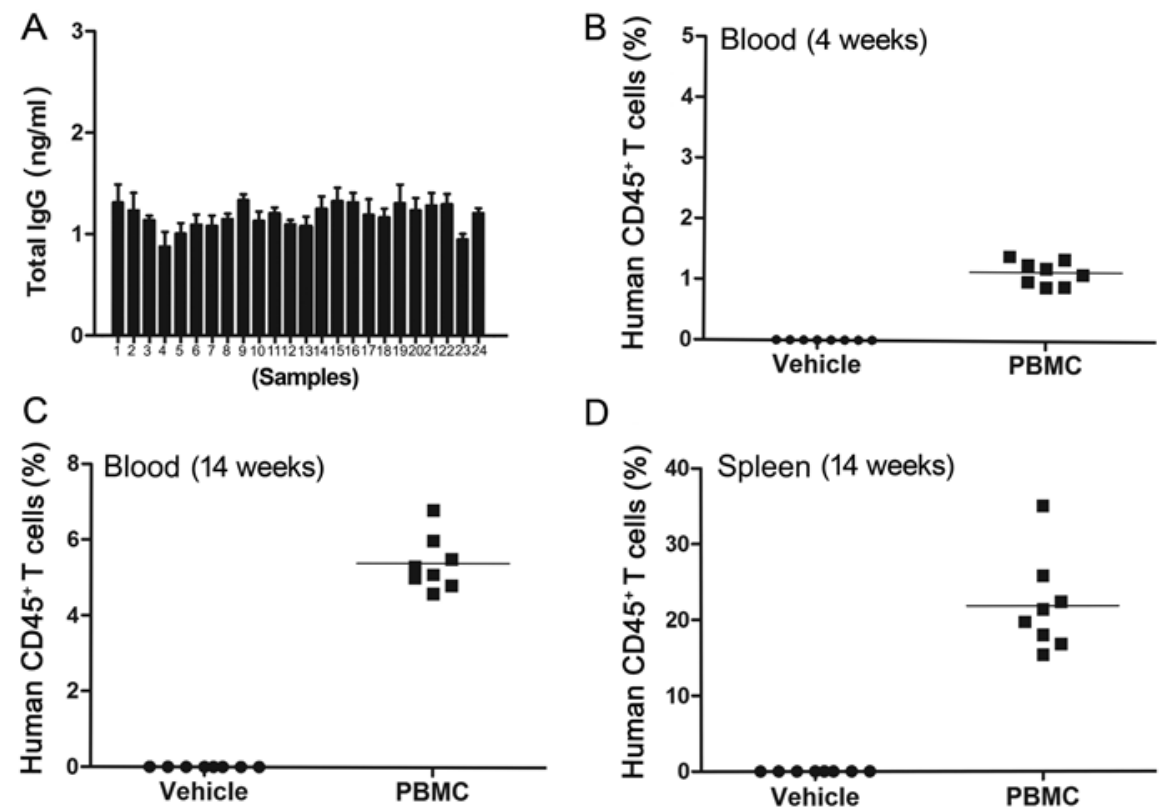

Figure 1. Establishment of the humanized mouse model. (A) Total murine serum immunoglobulin G was evaluated in NOD-SCID mice by ELISA. Non-leaky NOD-SCID mice were injected with PBS or human PBMCs $\left(4 \times 10^{7}\right.$ cells/mouse). Engraftment of human CD45 ${ }^{+}$T-lymphocytes in the peripheral blood was assessed by flow cytometry after (B) 4 and (C) 14 weeks. (D) Engraftment of human CD45 $5^{+}$-lymphocytes in the spleen were evaluated by flow cytometry after 14 weeks. Each circle represents an individual mouse. PBMCs, peripheral blood mononuclear cells; CD45, cluster of differentiation 45.
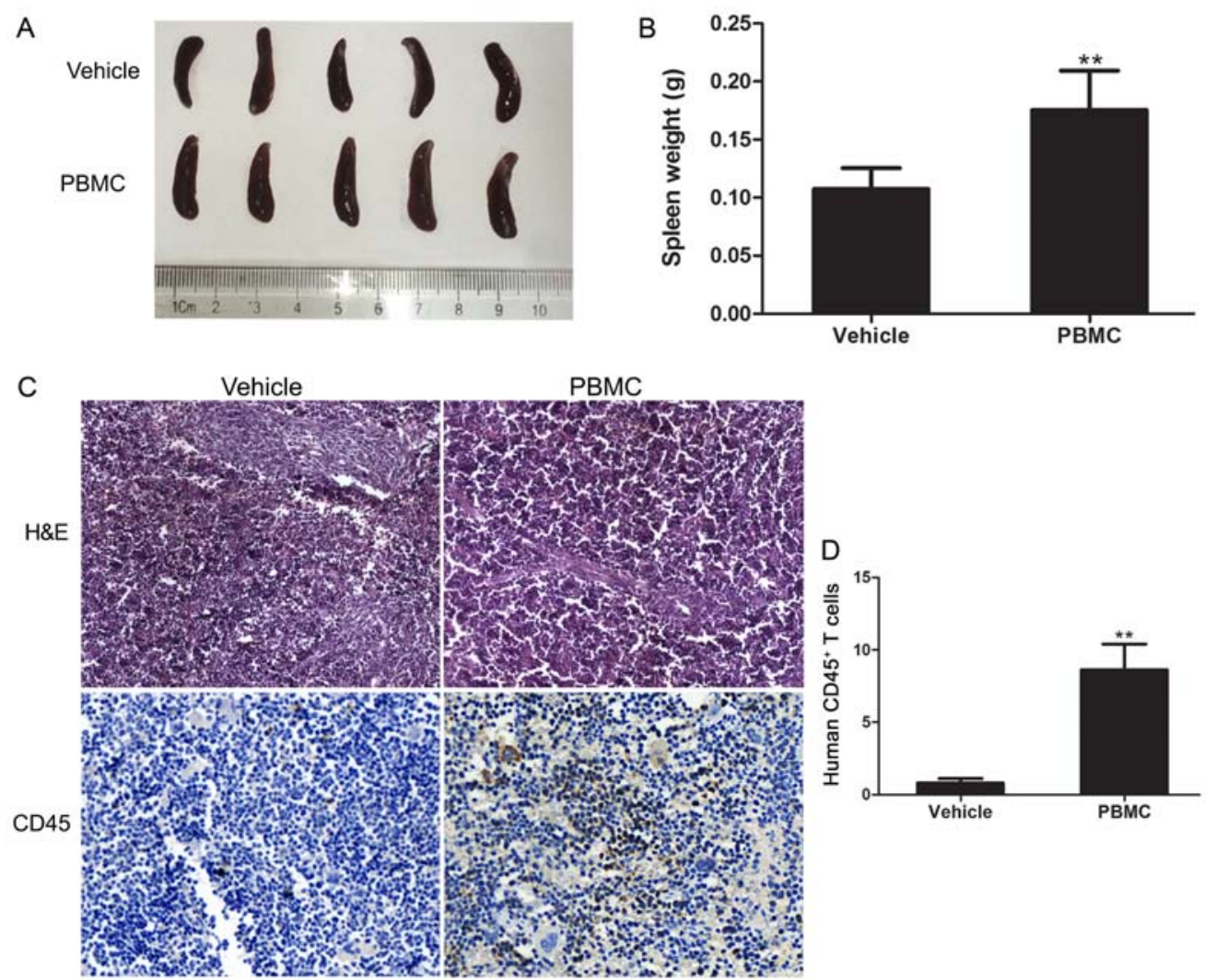

Figure 2. T-lymphocyte infiltration in the spleens of the model mice. (A and B) NOD-SCID mice were sacrificed 14 weeks after the engraftment of human PBMCs; the size and weight of splenic tissues were then measured. (C) Spleen tissue sections were excised from the mice, and the expression of CD45 was detected by immunohistochemical staining. (D) Quantification analysis of the CD45 levels in the splenic tissues. ${ }^{* *} \mathrm{P}<0.01$. PBMCs, peripheral blood mononuclear cells; CD45, cluster of differentiation 45.

signals $(\mathrm{P}<0.01$; Fig. $2 \mathrm{C}$ and $\mathrm{D})$. In conclusion, the results indicated that the humanized mouse model was established successfully.
HUVEC vaccine inhibits the angiogenesis of ESCCs in the humanized mouse model. A previous study demonstrated that immunization with the HUVEC vaccine prevented tumor 
A

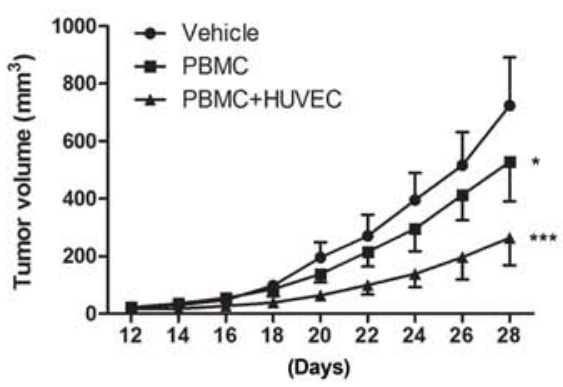

C

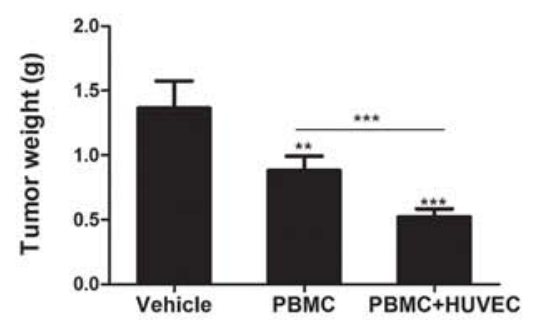

B

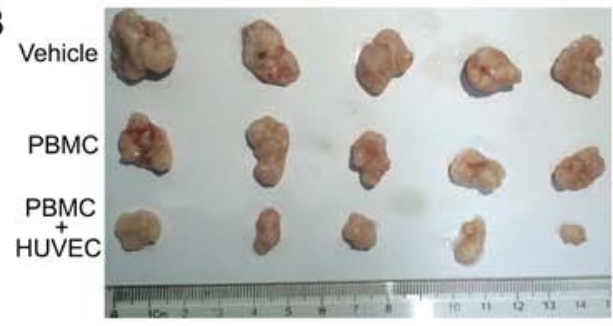

D

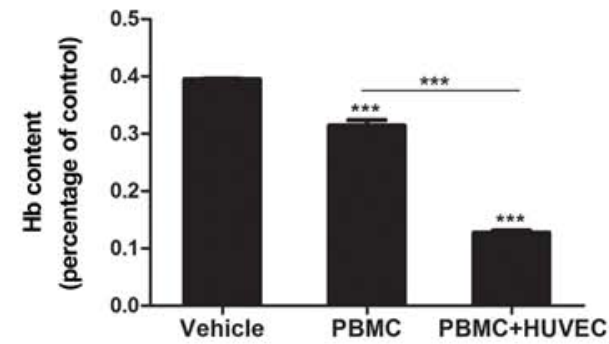

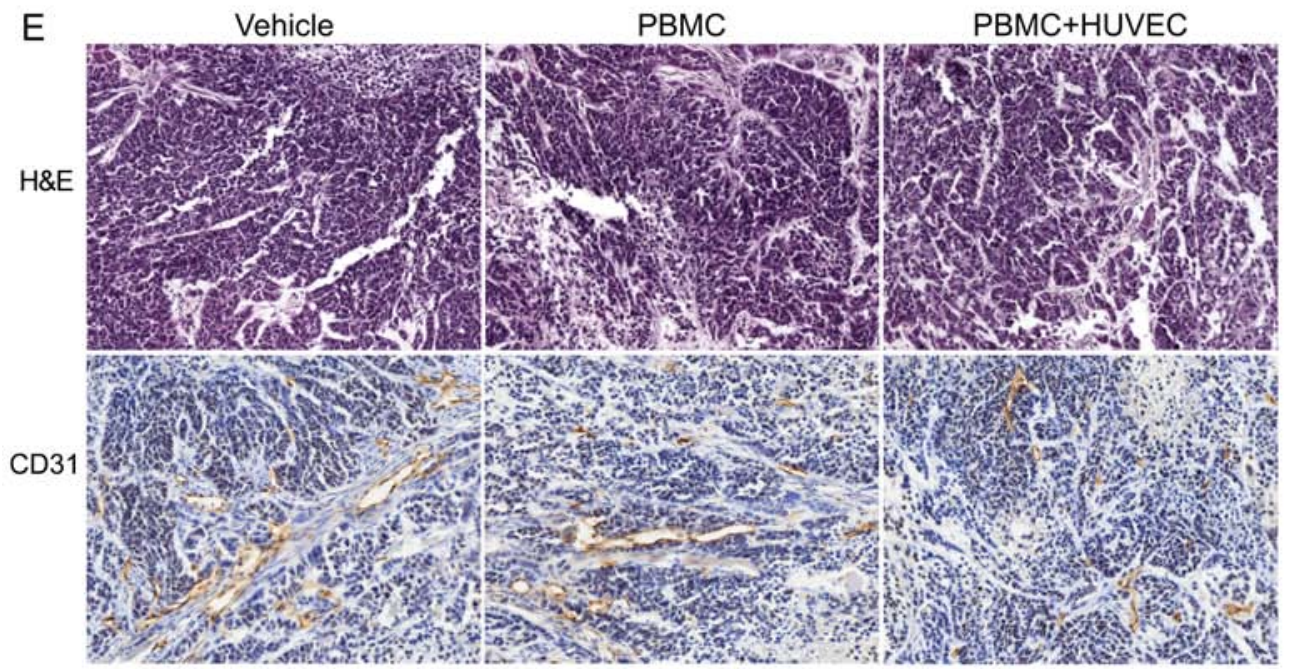

$\mathrm{F}$

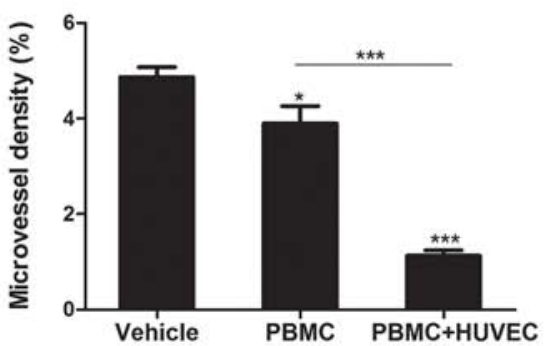

Figure 3. Humanized mice immunized with the HUVEC vaccine have inhibited ESCC growth via the suppression of tumor angiogenesis. Humanized mice were vaccinated with the HUVEC vaccine $\left(5 \times 10^{6}\right.$ cells/mouse) or PBS for 5 consecutive weeks. Then the mice were injected with EC 9706 cells $\left(6 \times 10^{6}\right.$ cells/mouse) subcutaneously. (A) The tumor volume was measured with calipers every other day from day 12 post-tumor cell challenge. (B and C) All of the mice were sacrificed 28 days after tumor initiation. Solid tumors were harvested for (B) imaging and (C) weighing. (D) Quantification analysis of the hemoglobin levels in the tumor tissues. (E) Immunohistochemical staining of vascularization was analyzed with an anti-CD31 antibody in tumor tissue sections. Random fields were analyzed. (F) The microvessel density was calculated. ${ }^{*} \mathrm{P}<0.05,{ }^{* *} \mathrm{P}<0.01$ and ${ }^{* * *} \mathrm{P}<0.001$. HUVEC, human umbilical vein endothelial cell; ESCC, esophageal squamous cell carcinoma; CD45, cluster of differentiation 45.

development and metastasis by utilizing the immune system to attack tumor vasculature in myeloma tumor models and Lewis lung carcinoma (17). However, there are increasing concerns regarding the value of this mouse model when translating the research to clinical application (18). Thus, an increasing number of studies have employed humanized mice to study human malignancies and biological processes (19-21).
In the present study, the humanized mice were vaccinated weekly with either PBS or the HUVEC vaccine for 5 weeks. Then, esophageal carcinoma EC9706 cells were implanted, measured over time and then the resulting tumors were removed for analyses. When compared with the vehicle group, tumor size was reduced in the PBMC group $(\mathrm{P}<0.05)$ and the PBMC + HUVEC group $(\mathrm{P}<0.001$; Fig. 3A). Notably, when 
A

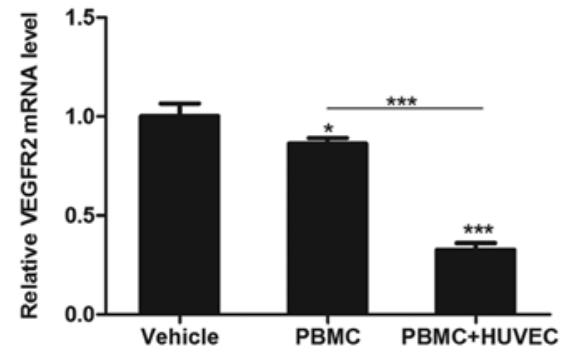

C

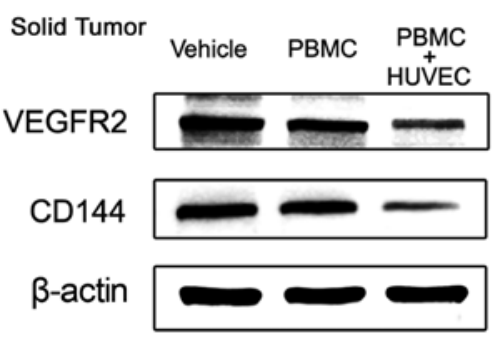

E
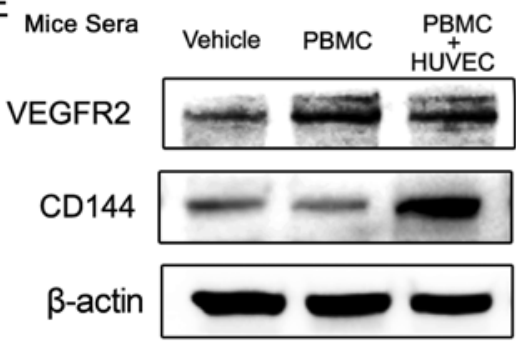

G

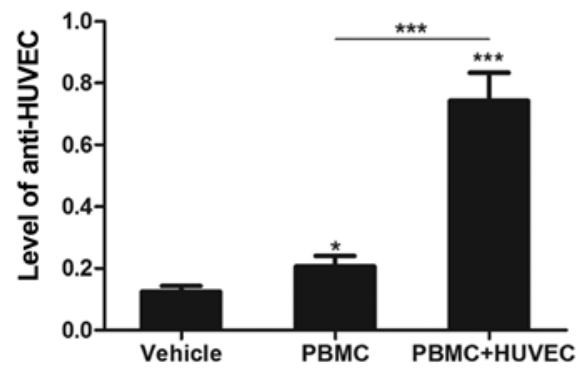

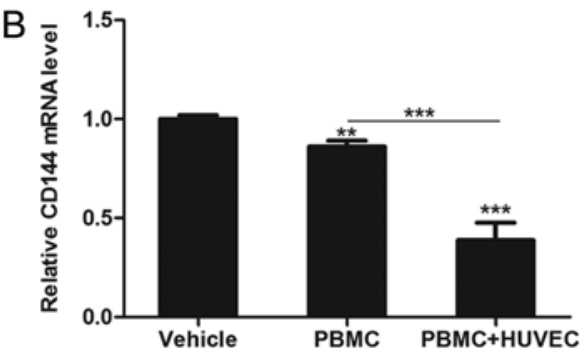

$\mathrm{D}$

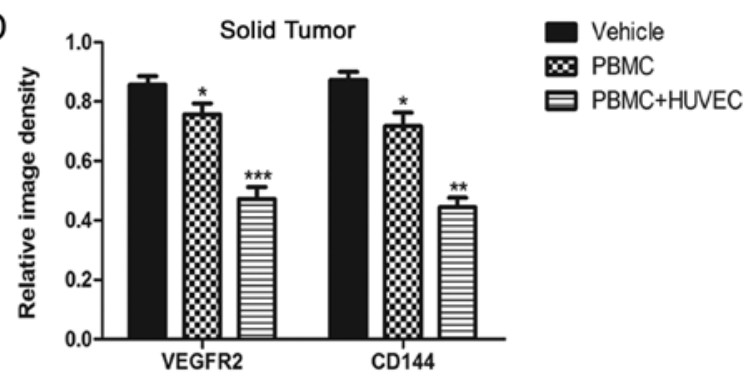

$\mathrm{F}$

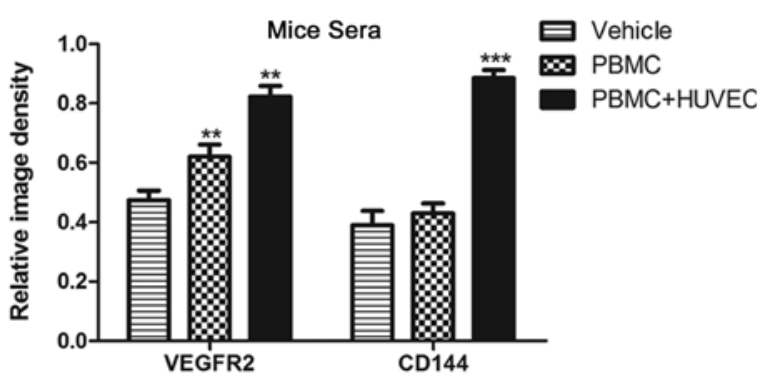

Figure 4. Expression of the angiogenesis-associated antigens and antibodies, VEGFR2 and CD144, in solid tumors and mice sera. (A and B) The mRNA levels of VEGFR2 and CD144 in tumors were detected by reverse transcription-quantitative polymerase chain reaction. (C) The protein levels of VEGFR2 and CD144 in solid tumors were analyzed by western blotting. (D) Quantification analysis of the VEGFR2 and CD144 protein levels in solid tumors. (E) The antibodies in the sera were evaluated by western blotting. (F) Quantification analysis of the VEGFR2 and CD144 antibody levels in serum. (G) The serum anti-HUVEC antibody levels were detected by ELISA. ${ }^{*} \mathrm{P}<0.05,{ }^{* *} \mathrm{P}<0.01$ and ${ }^{* * *} \mathrm{P}<0.001$. HUVEC, human umbilical vein endothelial cell; VEGFR2, vascular endothelial growth factor receptor 2; CD144, cluster of differentiation 144.

compared with the PBMC group, tumor size was significantly suppressed in the PBMC + HUVEC group $(\mathrm{P}<0.001$; Fig. 3B and C).

The present study then assessed the response of the HUVEC vaccine by analyzing angiogenesis in the tumor specimens. The hemoglobin assay revealed that the hemoglobin content in the PBMC + HUVEC group was significantly less than in the two other groups $(\mathrm{P}<0.001$; Fig. 3D). In addition, immunohistochemistry was conducted to investigate CD31 staining (an angiogenesis marker), and to analyze the MVD. The MVD in the PBMC+HUVEC group was significantly less than that observed in the vehicle and PBMC groups $(\mathrm{P}<0.001$; Fig. 3E and F). These results indicated that mice immunized with HUVECs may have inhibited ESCC growth via the suppression of tumor angiogenesis in the humanized mouse model.

Detection of antigens and antibodies in solid tumors and serum. To evaluate angiogenesis-associated antigens and antibodies in mice, the expression of VEGFR 2 and CD144 in solid tumors and serum was detected. After 4 weeks of tumor development, all of the mice were sacrificed, and the tumors and serum samples obtained were analyzed for mRNA and protein expression. The results of RT-qPCR indicated that the mRNA levels of VEGFR2 and CD144 in the solid tumors of the PBMC + HUVEC group were significantly reduced when compared with the two other groups $(\mathrm{P}<0.01$; Fig. 4A and B). The levels of VEGFR2 and CD144 proteins 
A

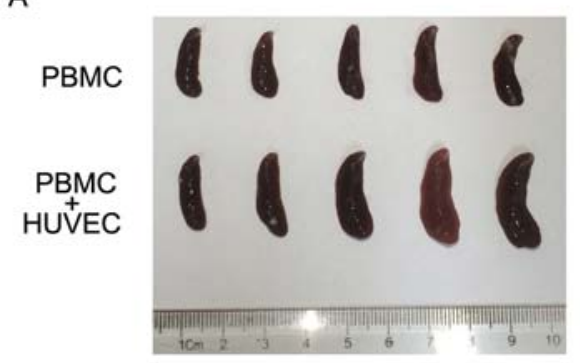

B

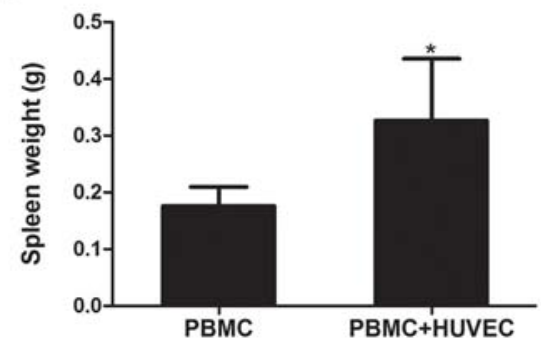

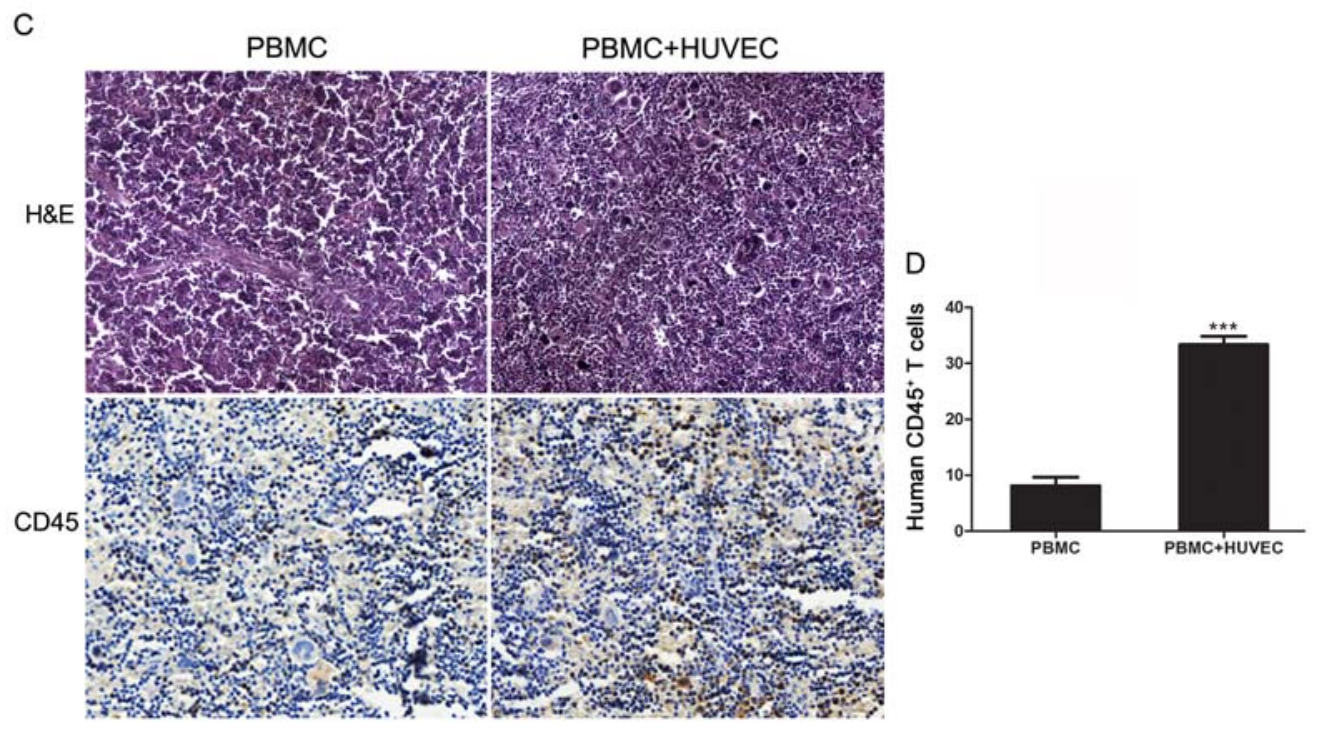

Figure 5. Immunization with the HUVEC vaccine increases T-lymphocyte infiltration into the spleens of humanized mice. (A and B) The mice were sacrificed 4 weeks after the last immunization with the HUVEC vaccine. The spleens were harvested, and the (A) size and (B) weight were measured. (C) The expression of CD45 in splenic tissues was detected by immunohistochemical staining. (D) Quantification analysis of the CD45 levels of splenic tissues. * $<0.05$, and ${ }^{* * * *} \mathrm{P}<0.001$. HUVEC, human umbilical vein endothelial cell; CD45, cluster of differentiation 45 .

in the solid tumors of the PBMC + HUVEC group were also significantly reduced $(\mathrm{P}<0.01$; Fig. $4 \mathrm{C}$ and $\mathrm{D})$. Mouse sera was also investigated using western blotting; and the results revealed that the serum antibody levels were significantly increased in the PBMC + HUVEC group $(\mathrm{P}<0.01$; Fig. 4E and F). In order to investigate the levels of the anti-HUVEC antibody in the serum of mice, ELISA analysis was performed, and the levels in mice immunized with the HUVEC vaccine were more marked $(\mathrm{P}<0.001 ;$ Fig. $4 \mathrm{G})$. The results demonstrated that the HUVEC vaccine increased the levels of angiogenesis-associated antigens in humanized mice.

Immunization with the HUVEC vaccine increases T-lymphocyte recruitment in the spleen. In order to evaluate the immune responses elicited by the HUVEC vaccine, the present study detected T-lymphocyte recruitment in mice after the last immunization. The weight and volume of the spleen were increased in the PBMC+HUVEC group ( $\mathrm{P}<0.05$; Fig. $5 \mathrm{~A}$ and $\mathrm{B})$. The results of splenic immunohistochemical staining demonstrated that the levels of human $\mathrm{CD}^{+} 5^{+} \mathrm{T}-$-lymphocytes in the PBMC + HUVEC group were significantly higher than in the PBMC group $(\mathrm{P}<0.001$; Fig. 5C and D). Overall, the results indicated that mice immunized with the HUVEC vaccine had increased T-lymphocyte recruitment in the spleens of humanized mice.

\section{Discussion}

A number of animal models have been developed and used in basic tumor research. Due to the technical and ethical considerations, investigations into the immunobiology of allotransplantation can only be performed using animals, which are limited when translating the results to humans (22). Progress has been made with the development of different chimeric animal models, which can serve important roles in studies associated with cancer, transplantation biology, infectious diseases, and autoimmunity (23). In the present study, human PBMC were engrafted into immunodeficient hosts, established mice with human lymphocytes, to characterize the effects of the HUVEC vaccine on esophageal carcinoma (16). Based on previous studies, mice were transplanted with PBMCs at a dose of $4 \times 10^{7} /$ mouse, which has been shown to have an increased success rate of immune reconstruction and a reduced risk of graft vs. host disease $(16,20,24)$. Among the various transplantation methods, the present study engrafted human PBMCs into NOD/SCID mice via an intraperitoneal injection because the method is more readily manipulated (22). After 4 weeks of immunization with human PBMCs, human $\mathrm{CD}^{4} 5^{+} \mathrm{T}$-lymphocytes were detected in mice. In addition, the weight of the spleens in the PBMC group was greater than in the vehicle group. The present study demonstrated the existence of T-lymphocytes to further assess the engraftment 
model. As a result, a large number of lymphocytes were identified in the spleen.

Angiogenesisis a vital part of sustained tumor development and progression, and involves comprehensive crosstalk among stromal cells, pericytes, tumor cells and vascular endothelial cells $(4,25)$. Therefore, inhibition of angiogenesis is currently being studied as a new anticancer therapy. Since the first vaccine targeting tumor angiogenesis was reported, proliferative HUVECs as a vaccine to induce antitumor immunity has been studied in several in vivo tumor models $(5-8,17,26)$. However, further investigation is required before the HUVEC vaccine can be used clinically, and the antitumor effect has not been studied previously in esophageal carcinoma. In the present study, the humanized mouse model immunized with the HUVEC vaccine significantly suppressed the size of the tumor. To further explore the underlying mechanism, the hemoglobin content and MVD of tumor specimens were evaluated. The results revealed that mice immunized with the HUVEC vaccine had reduced ESCC development due to suppression of tumor angiogenesis.

VE-Cadherin (CD144), an endothelial-specific adhesion protein that serves as an endothelial marker, mediates vascular stability and angiogenesis (27-29). VEGFR2, an overexpressed angiogenesis-associated antigen is appropriate for targeting the tumor vasculature via anti-angiogenic therapy, and is perceived to be a specific endothelial molecule (30). The present study detected the mRNA and protein levels of angiogenesis-associated antigens in the tumor specimen. It was demonstrated that the HUVEC vaccine decreased the levels of angiogenesis-associated antigens. The expression of antibodies and the ability of anti-HUVEC cells to proliferate were then evaluated in vitro in the serum from immunized mice. The results revealed that there were higher levels of antibodies in the sera of mice immunized with the HUVEC vaccine.

A critical role of the immune system is to suppress the progression of tumors and inhibit tumor growth. To further demonstrate that the HUVEC vaccine can induce these specific cellular immune responses, the present study monitored the levels of T-lymphocyte infiltration in the spleens. The weight of the spleens in the PBMC+HUVEC group was significantly higher than that observed in the PBMC group. Moreover, immunohistochemical staining indicated that a high level of human T-lymphocytes infiltrated the spleens of the PBMC+HUVEC group, which provided evidence that the HUVEC vaccine successfully induced immune responses in the humanized mouse model.

In conclusion, the present study revealed that the HUVEC vaccine inhibited ESCC growth by reducing tumor angiogenesis in a humanized mouse model. Thus, the results of the present study provide a potential model for evaluating immunotherapeutic approaches and highlights the potential use of HUVECs.

\section{Acknowledgements}

Not applicable.

\section{Funding}

The present study was supported by the National Natural Science Foundation of China (grant no. 81572972), the Science and Technology Innovation Talents Support Plan of University in Henan Province (grant no. 15HASTIT038) and the Colleges and Universities in Henan Province Key Scientific Research Plan (grant no. 15A310030).

\section{Availability of data and materials}

The datasets used during the present study are available from the corresponding author upon reasonable request.

\section{Author's contributions}

JL and HL conceived and designed the study and the experiments. HL, JZ, YY, GJ, XZ, DW, CX, KL, XC, XL and $\mathrm{FT}$ performed the experiments and analyzed the data. HL, YY and JL drafted, wrote or revised the manuscript. All authors read and approved the manuscript and agreed to be accountable for all aspects of the research in ensuring that the accuracy or integrity of any part of the work are appropriately investigated and resolved.

\section{Ethics approval and consent to participate}

All mice used in the study were treated and observed according to the guidelines of the Zhengzhou University Animal Ethics Committee (Henan, China).

\section{Patient consent for publication}

Not applicable.

\section{Competing interests}

The authors declare that they have no competing interests.

\section{References}

1. Zhang Y: Epidemiology of esophageal cancer. World J Gastroenterol 19: 5598-5606, 2013

2. Pujol JL, De Pas T, Rittmeyer A, Vallieres E, Kubisa B, Levchenko E, Wiesemann S, Masters GA, Shen R, Tjulandin SA, et al: Safety and immunogenicity of the PRAME cancer immunotherapeutic in patients with resected non-small cell lung cancer: A phase I dose escalation study. J Thorac Oncol 11: 2208-2217, 2016.

3. Yang Y, Lu J, Liu H, Jin G, Bai R, Li X, Wang D, Zhao J, Huang Y, Liu K, et al: Dendritic cells loading autologous tumor lysate promote tumor angiogenesis. Tumour Biol 37: 15687-15695, 2016.

4. Weis SM and Cheresh DA: Tumor angiogenesis: Molecular pathways and therapeutic targets. Nat Med 17: 1359-1370, 2011.

5. Xu M, Xing Y, Zhou L, Yang X, Yao W, Xiao W, Ge C, Ma Y, Yang J, Wu J, et al: Improved efficacy of therapeutic vaccination with viable human umbilical vein endothelial cells against murine melanoma by introduction of OK432 as adjuvant. Tumour Biol 34: 1399-1408, 2013.

6. Tanaka M, Tsuno NH, Fujii T, Todo T, Saito N and Takahashi K: Human umbilical vein endothelial cell vaccine therapy in patients with recurrent glioblastoma. Cancer Sci 104: 200-205, 2013.

7. Mu X, Sang Y, Fang C, Shao B, Yang L, Yao K, Zhao X, Gou J, Wei Y, Yi T, et al: Immunotherapy of tumors with human telomerase reverse transcriptase immortalized human umbilical vein endothelial cells. Int J Oncol 47: 1901-1911, 2015.

8. Xu M, Zhou L, Zhang P, Lu Y, Ge C, Yao W, Xing Y, Xiao W, Dong Y, Wu J, et al: Enhanced antitumor efficacy by combination treatment with a human umbilical vein endothelial cell vaccine and a tumor cell lysate-based vaccine. Tumour Biol 34: 3173-3182, 2013. 
9. Ellis LM and Fidler IJ: Finding the tumor copycat. Therapy fails, patients don't. Nat Med 16: 974-975, 2010.

10. Sanmamed MF, Rodriguez I, Schalper KA, Onate C, Azpilikueta A, Rodriguez-Ruiz ME, Morales-Kastresana A, Labiano S, Perez-Gracia JL, Martín-Algarra S, et al: Nivolumab and urelumab enhance antitumor activity of human T lymphocytes engrafted in $\mathrm{Rag} 2^{-/}$IL $2 \mathrm{R} \gamma^{\text {null }}$ immunodeficient mice. Cancer Res 75: 3466-3478, 2015

11. Theocharides AP, Rongvaux A, Fritsch K, Flavell RA and Manz MG: Humanized hemato-lymphoid system mice. Haematologica 101: 5-19, 2016.

12. Liu X, Li H, Liu J, Guan Y, Huang L, Tang H and He J: Immune reconstitution from peripheral blood mononuclear cells inhibits lung carcinoma growth in NOD/SCID mice. Oncol Lett 8: $1638-1644,2014$.

13. Banuelos SJ, Shultz LD, Greiner DL, Burzenski LM, Gott B, Lyons BL, Rossini AA and Appel MC: Rejection of human islets and human HLA-A2.1 transgenic mouse islets by alloreactive human lymphocytes in immunodeficient NOD-scid and NOD-Rag1 null Prf1 ${ }^{\text {null }}$ mice. Clin Immunol 112: 273-283, 2004.

14. Lu J, Zhao J, Liu K, Zhao J, Yang H, Huang Y, Qin Z, Bai R, Li P, Ma J, et al: MAPK/ERK1/2 signaling mediates endothelial-like differentiation of immature DCs in the microenvironment of esophageal squamous cell carcinoma. Cell Mol Life Sci 67: 2091-2106, 2010.

15. Jin G, Zhao J, Yang YI, Liu K, Jiang Y, Zhang X, Zhang Y, Huang Y, Lu J and Dong Z: JAK/STAT3 signaling pathway mediates endothelial-like differentiation of immature dendritic cells. Oncol Lett 10: 3471-3477, 2015.

16. King M, Pearson T, Shultz LD, Leif J, Bottino R, Trucco M, Atkinson MA, Wasserfall C, Herold KC, Woodland RT, et al: A new Hu-PBL model for the study of human islet alloreactivity based on NOD-scid mice bearing a targeted mutation in the IL-2 receptor gamma chain gene. Clin Immunol 126: 303-314, 2008.

17. Chen XY, Zhang W, Zhang W, Wu S, Bi F, Su YJ, Tan XY Liu JN and Zhang J: Vaccination with viable human umbilical vein endothelial cells prevents metastatic tumors by attack on tumor vasculature with both cellular and humoral immunity. Clin Cancer Res 12: 5834-5840, 2006.

18. Holzapfel BM, Wagner F, Thibaudeau L, Levesque JP and Hutmacher DW: Concise review: Humanized models of tumor immunology in the 21st century: Convergence of cancer research and tissue engineering. Stem Cells 33: 1696-1704, 2015.
19. Brehm MA, Shultz LD and Greiner DL: Humanized mouse models to study human diseases. Curr Opin Endocrinol Diabetes Obes 17: 120-125, 2010

20. Shultz LD, Ishikawa F and Greiner DL: Humanized mice in translational biomedical research. Nat Rev Immunol 7: 118-130, 2007.

21. Shultz LD, Brehm MA, Garcia-Martinez JV and Greiner DL: Humanized mice for immune system investigation: Progress, promise and challenges. Nat Rev Immunol 12: 786-798, 2012.

22. Gong Z, Xu H, Su Y, Wu W, Hao L and Han C: Establishment of a novel bladder cancer xenograft model in humanized immunodeficient mice. Cell Physiol Biochem 37: 1355-1368, 2015.

23. Yacoub-Youssef H, Marcheix B, Calise D, Thiers JC, Therville N, Benoist H, Blaes N, Segui B, Dambrin C and Thomsen M: Engraftment of human T, B and NK cells in CB.17 SCID/beige mice by transfer of human spleen cells. Transpl Immunol 15: 157-164, 2005.

24. Ji M, Jin X, Phillips P and Yi S: A humanized mouse model to study human immune response in xenotransplantation. Hepatobiliary Pancreat Dis Int 11: 494-498, 2012.

25. Folkman J: Angiogenesis: An organizing principle for drug discovery? Nat Rev Drug Discov 6: 273-286, 2007.

26. Wei YQ, Wang QR, Zhao X, Yang L, Tian L, Lu Y, Kang B, Lu CJ, Huang MJ, Lou YY, et al: Immunotherapy of tumors with xenogeneic endothelial cells as a vaccine. Nat Med 6: 1160-1166, 2000 .

27. PeifferDS,Zimmerman NP, Wang LS, Ransom BW, Carmella SG, Kuo CT, Siddiqui J, Chen JH, Oshima K, Huang YW, et al: Chemoprevention of esophageal cancer with black raspberries, their component anthocyanins, and a major anthocyanin metabolite, protocatechuic acid. Cancer Prev Res 7: 574-584, 2014

28. Dejana E and Giampietro C: Vascular endothelial-cadherin and vascular stability. Curr Opin Hematol 19: 218-223, 2012.

29. Vestweber D: VE-cadherin: The major endothelial adhesion molecule controlling cellular junctions and blood vessel formation. Arterioscler Thromb Vasc Biol 28: 223-232, 2008.

30. Okaji Y, Tsuno NH, Saito S, Yoneyama S, Tanaka M, Nagawa $H$ and Takahashi K: Vaccines targeting tumour angiogenesis-a novel strategy for cancer immunotherapy. Eur J Surg Oncol 32: 363-370, 2006. 